\title{
Ai Distress Beacon using Speech Recognition
}

\author{
Arman Shaikh $^{1}$, Mutasim Rehman ${ }^{2}$, Rushikesh Mate ${ }^{3}$, Ritesh Ghate ${ }^{4}$, Prof. Mrs. A.S. Khandagale ${ }^{5}$ \\ Student, Department of Computer Engineering All India Shri Shivaji Memorial Society Polytechinc, Kennedy road, \\ Pune, Maharashtra, India ${ }^{1,2,3,4}$ \\ Lecturer, Department of Computer Engineering All India Shri Shivaji Memorial Society Polytechnic Kennedy Road, \\ Pune, Maharashtra, India ${ }^{5}$

\begin{abstract}
In recent time, there are many misbehaving activity happening with women in urban and rural areas. This paper is about 'An Android Application for Women Safety Based on Voice Recognition'. One in which, keywords of different languages are set for activation of the system. System recognizes the voice and frequency of voice of the user. After system gets activate, it uses a GPS or WI-FI to track the location information of the user and send that location information as a message via SMS and post it on Social Application. Simultaneously, an emergency signal is sent to people who are selected by the mobile user. There is no need to handle mobile to activate the system and it will get activated even if mobile keypad is locked.
\end{abstract}

Keywords: Android, Voice Recognition, Dial 100, Messagesending, GPS, WI-FI, HMM, Audio Recording.

\section{INTRODUCTION}

Women safety is major issue in India and also in other countries. It is not safe for women to travel lonely at mid night or wondering in unknown place. There should be helping hand for women because they are not physically as strong as men. As this time mobile phone can be best friend of the user. User can stay in contact with their loved one at any time. Anybody needs to make a call or send a message in emergency at anytime from anywhere.

Smart phones provides many facilities and cheaper internet cost. Android is provided with features like high resolution camera, Wi-Fi, GPS navigation, touch screen which helps the mobile phone users to keep in touch with the modern world. Android is a software stack for mobile devices. It includes an operating system, key applications and middleware. The Android SDK provides the tools and APIs use to develop applications on the Android platform using the Java programming language. It is a software platform, rather than just an OS, that has the potential to be utilized in a much wider range of devices. Android provides access to useful libraries and tools that used to build rich applications. Women in emergency uses voice based contact list, they can select contact through voice and make call when required. For that user have to remember registered the key. It is called, text messaging service component of the phone, and uses standardized communications protocols. It allows sending of short text messages between mobile phone devices. Speech Recognition and Conversion in text will be the necessary part of the system.

\section{IMPLEMENTATION AND DESIGNING}

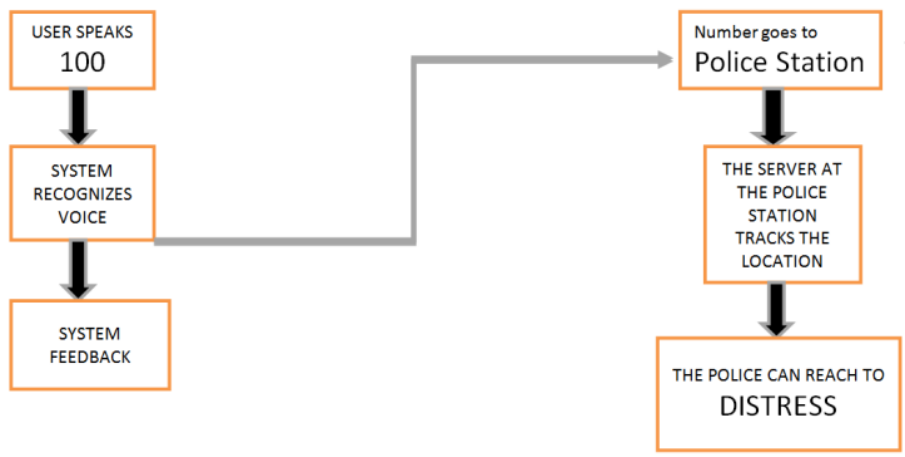

Fig 1.MODULE DESCRIPTION

Voice recognition module is use to recognize keyword spoken by the user. It requires voice recorder to store voice in mobile phone. Hidden Markov Model is used for voice recognition and it converts voice to text. Voice keywords are stored in mobile memory. This keyword is matched with converted text. 


\section{EXISTING SYSTEM:}

Now we now that girls' safety is of prior importance in today's world. There is no such system which can provide the safety regarding girls and therefore the girl indirectly loses her right to social freedom. There are different products for the girls' safety, such as shoes, aerosol sprays, etc. But these cannot provide safety in the adverse conditions. There are also certain smartphone apps for tackling such situations, but they are still not so much responsive and popular in the outside world.

\section{Advantage:}

The distress person doesn't have to manually execute the commands, he just has to speak the command and the location will be reached to the nearby police station,thus reducing the time.

\section{Proposed System:}

The current women safety applications in market have to push the button to send message. It is difficult to press button in critical situation when keypad is locked. This paper deals with recognizing voice converted into text to send message. Message consists of GPS location information of the user. Message can be send even if the keypad is locked. a.Voice recognition module is use to recognize keyword spoken by the user. It requires voice recorder to store voice in mobile phone. Hidden Markov Model is used for voice recognition and it converts voice to text [6]. Voice keywords are stored in mobile memory. This keyword is matched with converted text.

\begin{tabular}{|l|}
\hline Voice Recognition \\
\hline \\
\hline Voice to Text Conversion \\
\hline \\
\hline Message Sending \\
\hline
\end{tabular}

\section{DESIGN}

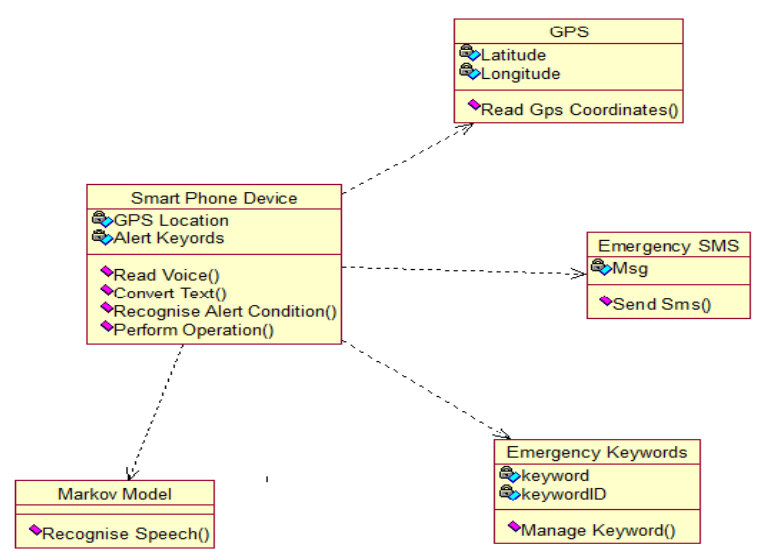

Fig 3.Class diagram

The class diagram is the main building block of object-oriented modelling it is used for both general conceptual modelling of the application of the systematics of the applications, and for detailed modelling translating the models into programming code. Class diagrams can be also used for data modelling. In the above class diagram the description of the various classes and their functions is shown.

\section{Requirement Analysis}

\section{Hardware Requirement}

- An android smartphone

- Laptop which will act as a Server (Police Station)

\section{Software Requirement}

- Android Studio

- Android SDK Tools

- NetBeans 


\section{IJARCCE}

- Apache Tomcat

- MySQL Workbench

\section{CONCLUSION}

Through this paper successfully concludes that the human voice interaction with the device (machine) is effectively utilized to tackle hazardous problem of crime in the world. The amount of crimes occurring on a daily basis to mankind can be drastically reduced and we can make the world a safe place to live in. This device will work instantly without any delay and much faster than the mobile phones.

\section{REFERENCES}

[1] Dongare Uma, VyavahareVishakha and RautRavina, “An Android Application for Women Safety Based on Voice Recognition”, Department of Computer Sciences BSIOTR wagholi, SavitribaiPhule Pune University India, ISSN 2320-088X International Journal of Computer Science and Mobile Computing (IJCSMC) online at www.ijcsmc.com,Vol.4 Issue.3, pg. 216-220, March- 2015

[2] MAGESH KUMAR.S and RAJ KUMAR.M, "IPROB -EMERGENCY APPLICATION FOR WOMEN", Department of Computer science Sree Krishna College of Engineering Unai village Vellore (TN) India, ISSN 2250-3153 International Journal of Scientific and Research Publications, online at the link www.ijsrp.org, Volume 4, Issue 3, March 2014.

[3] VaijayantiPawar, Prof. N.R.Wankhade, DipikaNikam, KanchanJadhav and NehaPathak, "SCIWARS Android Application for Women Safety", Department of Computer Engineering, Late G.N.S.COE Nasik India, ISSN: 2248-9622 International Journal of Engineering Research and Applications Online at the link www.ijera.com, Volume 4, Issue 3(Version 1), pp.823826, March 2014. 